\title{
OPTIMAL BOUNDS FOR LINEAR FUNCTIONALS ON MONOTONE FUNCTIONS
}

\author{
TOMASZ RYCHLIK
}

Abstract. We determine optimal bounds on linear functionals over the space of square integrable functions on a finite interval, restricted to the nondecreasing elements of the subspace orthogonal to constants. We discuss conditions of bounds attainability and present exemplary applications.

Mathematics subject classification (2000): 26D15, 46E30.

Key words and phrases: $L^{2}$-space, linear functional, monotone function, optimal bound.

\section{REFERENCES}

[1] H. A. David, H. N. Nagaraja, Order Statistics, 3rd Ed., Wiley, Hoboken, NJ, 2003.

[2] N. DuNFORD, J. T. SCHWARZ, Linear Operators I, Wiley, New York, 1958.

[3] A. GoRONCY, T. RYCHLIK, How deviant can you be? The complete solution, Math. Inequal. Appl., 9, (4) (2006), 633-647.

[4] U. KAMPS, A Concept of Generalized Order Statistics, Teubner, Stuttgart, 1995.

[5] A. W. MARSHALl, I. OlKIN, Inequalities: Theory of Majorization and Its Applications, Academic Press, New York, 1979.

[6] D. S. Mitrinović, Analytic Inequalities, Springer-Verlag, Berlin, 1970.

[7] S. MoriguTI, A modification of Schwarz's inequality, with applications to distributions, Ann. Math. Statist., 24, (1953), 107-113.

[8] T. RYCHLIK, Sharp inequalities for linear combinations of elements of monotone sequences, Bull. Polish Acad. Sci. Math., 40, (1992), 247-254.

[9] T. RYCHLIK, Projecting Statistical Functionals, Lecture Notes in Statist., 160, Springer, New York, 2001. 